\title{
Real-world investigation on discontinuation of oral anticoagulation after paroxysmal atrial fibrillation catheter ablation in China
}

\author{
Ronghui Yu ${ }^{1,2}$, Hui Xi ${ }^{3}$, Jun $\mathrm{Lu}^{4}$, Fengqiang $\mathrm{Xu}^{4}$, Lisheng Shi ${ }^{5}$, Qiang Kong ${ }^{5}$, Yucai Hu ${ }^{6}$, Xin $\mathrm{Zhao}^{1,2}$, \\ Nian Liu ${ }^{1,2}$ \\ ${ }^{1}$ Department of Cardiology, Beijing Anzhen Hospital, Capital Medical University, Beijing 100029, China; ${ }^{2}$ National Clinical Research Center for \\ Cardiovascular Diseases, Beijing 100029, China; ${ }^{3}$ Department of Cardiology, Peking University International Hospital, Beijing 102206, China; \\ ${ }^{4}$ Department of Cardiology, The Affiliated Hospital of Qingdao University, Qingdao 266000, China; ${ }^{5}$ Department of Cardiology, Xuanwu Hospital, \\ Capital Medical University, Beijing 100053, China; ${ }^{6}$ The First Affiliated Hospital of Henan University of Traditional Chinese Medicine, Zhengzhou \\ 450000, China \\ Contributions: (I) Conception and design: R Yu; (II) Administrative support: X Zhao, N Liu; (III) Provision of study materials or patients: H Xi, J \\ Lu, F Xu, Q Kong, Y Hu; (IV) Collection and assembly of data: X Zhao, N Liu; (V) Data analysis and interpretation: R Yu, X Zhao, N Liu; (VI) \\ Manuscript writing: All authors; (VII) Final approval of manuscript: All authors. \\ Correspondence to: Ronghui Yu. Department of Cardiology, Beijing Anzhen Hospital, Capital Medical University, Beijing 100029, China. \\ Email: ronghuiyu20@163.com.
}

Background: To find out the real-world investigation on discontinuation of oral anticoagulation after paroxysmal atrial fibrillation (AF) catheter ablation China.

Methods: We enrolled in our study 1,508 consecutive paroxysmal AF patients who underwent catheter ablation from five centers. Patients' clinical data and follow-up data were collected. Clinical data included on-admission characteristics such as gender, age, type and duration of AF, type of ablation, ablation sessions, history of diseases, $\mathrm{CHA}_{2} \mathrm{DS}_{2}$-VASc score, echocardiographic variables, medication, and blood test variables. Follow-up data included duration of follow-up, the status of oral anticoagulant (OAC) therapy, adverse events, and recurrence of AF.

Results: A total of 1,491 patients were included in the final analysis, while the other 17 patients lost contact. The follow-up duration was 12 to 74 months (27.8 14.6 months). Of 1,491 patients, 989 (66.3\%) patients stopped OAC therapy 3 to 14 months after successful ablation during follow-up. Stroke or transient ischemic attack (TIA) occurred in 37 (2.5\%) patients. Major bleeding occurred in 24 (1.6\%) patients. Six $(0.4 \%)$ patients died at follow-up. Patients who stopped OAC had lower CHA2DS2-VASc score $(1.5 \pm 1.4$ vs. $2.4 \pm 1.7, \mathrm{P}<0.05)$ and lower incidence of major bleeding $(11 / 989$ vs. $13 / 502, \mathrm{P}<0.05)$. Of 989 patients who stopped OAC, 318 stopped according to doctor's prescription, and 671 stopped on their own decision. In patients who underwent brain MRI, patients who stopped OAC had a lower incidence of silent cerebral infarction (SCI) $(37 / 904$ vs. 38/419, $\mathrm{P}<0.05)$. Among patients who did not stop warfarin therapy, there were $117(117 / 397,29.5 \%)$ patients had not to achieve target INR.

Conclusions: The incidence of adverse events was relatively low in paroxysmal AF patients who stopped OAC. The patient partly determined when OAC was discontinued. Target INR was not achieved in many patients who had not to stop OAC.

Keywords: Oral anticoagulation; paroxysmal atrial fibrillation (paroxysmal AF); catheter ablation

Submitted Feb 10, 2020. Accepted for publication Mar 07, 2020.

doi: 10.21037/apm-20-565

View this article at: http://dx.doi.org/10.21037/apm-20-565 


\section{Introduction}

In China, the prevalence of incident AF in subjects aged $\geq 20$ years was 0.2 per 100 people, with an incidence of AF of 0.05 per 100 person-years, overall. Over 11 years, the prevalence of AF increased 20-fold, whereas AFrelated stroke increased 13-fold (1-3). Atrial fibrillation (AF) is correlated with some serious adverse cardiovascular events, such as stroke or transient ischemic attack (TIA), heart failure, and even death. Studies also demonstrated that AF decreases the quality of life and increases the risk of dementia or cognitive disability (4). Sinus rhythm recovery and maintenance are thought to be better for AF patients' prognosis compared with heart rate control alone (5). Radiofrequency catheter ablation (RFCA) has become the main method to acquire sinus rhythm. In recent years, China has entered the era of catheter ablation for AF patients. More and more patients with AF underwent RFCA, cryoballoon ablation, or hot balloon ablation (6). Oral anticoagulant (OAC) can reduce the risk of stroke/ TIA in AF patients and is continued after ablation for some days (7). Nevertheless, OAC can also increase the risk of major bleeding, especially cerebral bleeding, which is often vital (8). So, in clinical practice, OAC continuation is often a complicated question faced by both doctors and patients. In this study, we retrospectively analyzed real-world AF patients' follow-up data in China to summarize the actual status of OAC use after catheter ablation and relevant problems.

\section{Methods}

\section{Study population}

Patients underwent RFCA in five centers in China from Nov 2014 to Oct 2017 were enrolled according to inclusion criteria and exclusion criteria. Inclusive criteria: paroxysmal AF, successful RFCA, follow-up as planned, received repeated blood test, and examination at regular follow-up. Exclusive criteria: valvular heart disease, renal insufficiency, malignant tumor, pregnancy. All patients supplied written informed content. The ethics committee approved this study of each center. Patients were divided into two groups: a group of patients who stopped OAC during follow-up (SG) and a group of patients who continued to take OAC during follow-up (CG). Paroxysmal AF refers to AF that ends spontaneously and sustains less than 7 days $(6,7,9)$.

\section{Data collection}

Patients' information, including baseline characteristics, RFCA procedure, and follow-up data, were collected from in-hospital records, follow-up clinic records, and their reports. Baseline characteristics included gender, age, history of the disease, medication, on admission blood test including liver function, renal function, electrolyte, lipid, fast glucose, HbA1c, hs-CRP etc., and examination including echocardiography, 24 hours Holter monitoring and regular electrocardiogram, etc. RFCA procedure information included the duration of the procedure, time of fluoroscopy exposure, etc. Follow-up data included medication, repeated blood test especially PT, APTT and INR when OAC was administrated, repeated echocardiography, time from successful RFCA to discontinuation of OAC, adverse events including stroke/TIA, major bleeding, AF recurrence, heart failure, and death, etc. Patients' information of general group and subgroups, including SG and CG, were summarized, respectively.

\section{Catbeter ablation procedure}

All patients underwent catheter ablation radiofrequency. In these 5 centers, the procedure of catheter ablation was similar and conducted according to guidelines $(4,5)$ and all patients signed informed content. In short, amiodarone was stopped for at least 1 week before ablation, and other anti-arrhythmia drugs were stopped for at least five halflife periods. Transesophageal echocardiography was used to detect thrombosis in the left atrium before ablation. After local anesthesia with $1 \%$ lidocaine, $8.5 \mathrm{~F}$ sheath was implanted into the right femoral vein, and $6 \mathrm{~F}$ sheath was implanted into the left femoral vein. The mapping electrode was implanted into the coronary sinus through $6 \mathrm{~F}$ sheath. After atrial septum was punctured, a saline catheter (Thermo Cool Smart Touch, Biosense-Webster Inc., Diamond Bar, CA, USA) was moved into the left atrium. The radiofrequency ablation and 3-D model was conducted under the ThreeDimensional system (CARTO3, Biosense-Webster, Diamond Bar, CA, USA) guiding. Continuous circumferential ablation around the ipsilateral pulmonary vein cavity was performed to isolate all pulmonary veins completely.

\section{Follow-up}

All patients were followed up regularly at 1,3 , and 
12 months after the RFCA procedure. Twelve months after the RFCA procedure, patients were followed up annually. All follow-ups were conducted at each center's outpatient clinic. At 3 months follow-up, some patients' medication therapy was adjusted according to their condition and doctors' experience or guideline.

\section{Statistical analysis}

Continuous variables were expressed as the mean \pm standard deviation (SD) and compared using an unpaired twosided Student's $t$-test when normal distribution and equal dispersion were confirmed. The Mann-Whitney $U$ test and the Wilcoxon's signed-rank test were used when the variance was unequal. Categorical variables were expressed as percentages (\%) and compared using $\chi^{2}$ analysis or Fisher's exact test if necessary. Freedom from adverse events at follow-up was analyzed by Kaplan-Meier survival curves and compared by the log-rank test. A P value of less than 0.05 was considered statistically significant. All statistical analyses were performed with SPSS (version 17.0 for Windows, SPSS, Inc., Chicago, IL, USA).

\section{Results}

\section{Baseline characteristics in general group and subgroups}

According to inclusive and exclusive criteria, 1,491 patients were enrolled in the final analysis. In general, among 1,491 patients, 918 (61.6\%) patients were male, and 573 (38.4\%) patients were female. Patients' age ranged from 43 to 76 years old, and the mean age was $59.6 \pm 12.1$ years old. Of these 1,491 patients, $989(66.3 \%)$ patients stopped OAC therapy 3 months to 14 months after successful ablation during follow-up. Then patients were divided into two groups: a group of patients who stopped OAC during followup (SG) and a group of patients who continued to take OAC during follow-up (CG). Patients who stopped OAC had a lower $\mathrm{CHA}_{2} \mathrm{DS}_{2}$-VASc score $(1.5 \pm 1.4$ vs. $2.4 \pm 1.7, \mathrm{P}<0.05)$. Of 989 patients who stopped OAC, 411 patients' $\mathrm{CHA}_{2} \mathrm{DS}_{2}-$ VASc score was over 2, which showed a substantial risk of ischemic stroke/TIA. Details were listed in Table 1.

\section{Follow-up outcomes in SG and CG}

During follow-up, 274 (18.4\%) adverse events occurred with $151(15.3 \%)$ in SG and $123(24.5 \%)$ in CG, respectively. Stroke or TIA occurred in 37 (2.5\%) patients, but no difference existed between SG and CG. Patients in CG had a higher incidence of major bleeding compared with patients in SG (2.6\% vs. $1.1 \%, \mathrm{P}<0.05)$. Six $(0.4 \%)$ patients died at follow-up. Heart failure occurred in 48 patients and its incidence in CG was higher than in SG (5.8\% vs. $1.9 \%, \mathrm{P}=0.0001)$. There was no difference in the incidence of death $(3 / 989$ vs. 3/502, $\mathrm{P}>0.05)$ between SG and CG. Of patients who stopped OAC, 318 stopped according to doctors' orders, and 671 stopped on their own decision. In these patients, 411 had a $\mathrm{CHA}_{2} \mathrm{DS}_{2}$-VASc score over 2, of which 18 (4.4\%) developed stroke/TIA. During follow-up, 1,323 (88.7\%) patients underwent a brain MRI examination. In patients who underwent brain MRI, patients who stopped OAC had a lower incidence of silent cerebral infarction (SCI) $(37 / 904$ vs. 38/419, $\mathrm{P}<0.05)$. Among patients who did not stop warfarin therapy, there were 117 (117/397, 29.5\%) patients had not achieved target INR. For details, see Table 2. Kaplan-Meier Survival curves of freedom from adverse events in SG and CG are shown in Figure 1. Survival analysis revealed that patients in the SG had similar outcomes compared to patients in the CG $(\mathrm{P}>0.05)$.

\section{Discussion}

With the rapid progress of RFCA for AF patients in recent years, the number of AF patients who underwent RFCA increased significantly in China (8). Successful RFCA leads to the recovery of sinus rhythm, which has a better prognosis than heart rate control alone (10). However, whether post-ablation anticoagulant therapy is necessary or not is still controversial (11). Most guidelines or consensus suggest that patients at high risk of thrombosis events prior to RFCA procedure, i.e., CH2ADS2-VASc score over 2, should continue to take OAC after RFCA procedure for a long time or even lifelongKL. This strategy reduces the incidence of ischemic vascular events while increases the risk of minor and major bleeding, which is often lifethreatening. So, in clinical practice, what is the fact about OAC therapy after the RFCA procedure, especially in China? Our present study partly answered this question.

By retrospective analysis of data involving 1,491 paroxysmal AF patients from five centers in China, we found that over two-thirds (66.3\%) of patients stopped OAC either on doctors' prescriptions or on their own decision. This rate was higher than those in another study $(12,13)$. In their study, Okumura et al. reported $1,836(53 \%)$ patients discontinued OAC therapy during 
Table 1 Baseline characteristics in the whole group and subgroups

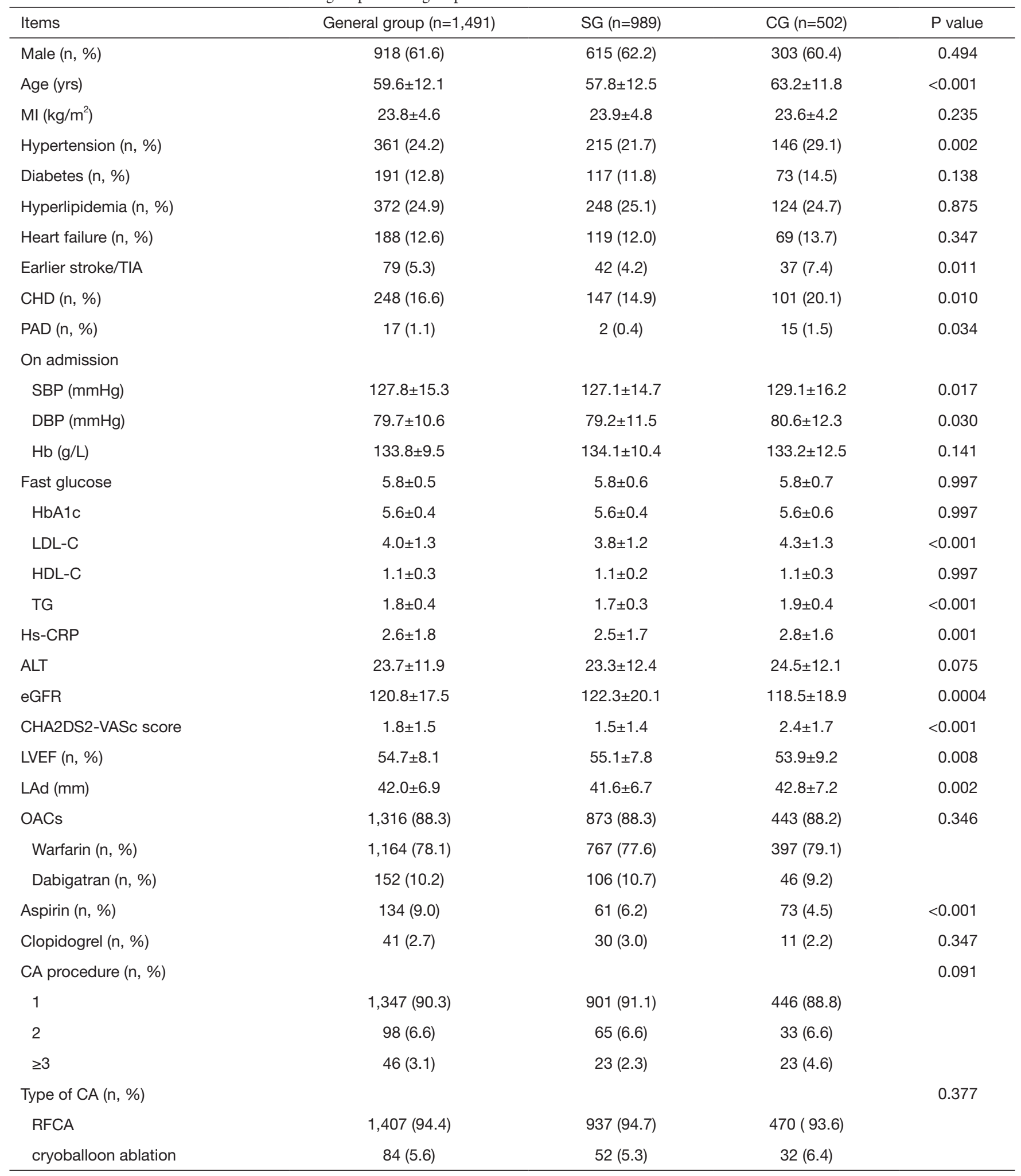

BMI, body mass index; CHD, coronary heart disease; PAD, peripheral artery disease; SBP, systolic blood pressure; DBP, diastolic blood pressure; LDL-C, low density lipoprotein cholesterol; HDL-C, high density lipoprotein cholesterol; TG, triglyceride; hs-CRP, high sensitivity $\mathrm{C}$ reaction protein; ALT, alanine aminotransferase; Cr, creatine; Lad, left atrium diameter; OAC, oral anticoagulant. 
Table 2 Follow-up outcomes

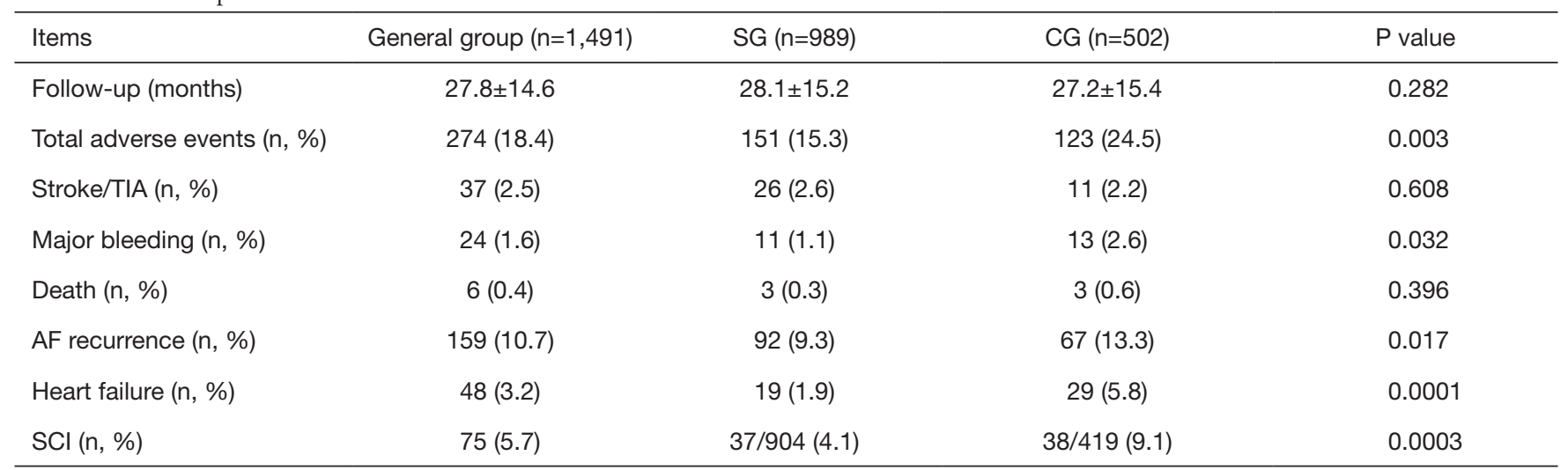

SG, group of patients who stopped OAC during follow-up; CG, group of patients who continued to take OAC during follow-up; TIA, transient ischemic attack; AF, atrial fibrillation; SCI, silent cerebral infarction.

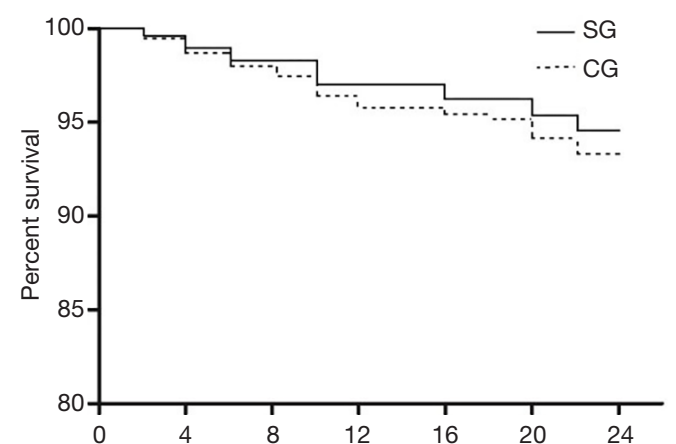

Figure 1 Kaplan-Meier survival curve of 24 months in SG and CG, $\mathrm{P}=0.084$. SG, group of patients who stopped OAC during follow-up; CG, group of patients who continued to take OAC during follow-up.

a 20.7-month follow-up period (12). They found that patients who stopped OAC therapy were mainly younger, male, paroxysmal AF, only a single AF session, absence of comorbidities, a smaller left atrium diameter (Lad), etc. We found that most patients who stopped OAC had a lower risk of stroke/TIA and similar prognosis compared with those continued to take OAC. In those who stopped OAC, some patients followed doctors' prescriptions, others decided by themselves. Doctors' decisions on discontinuation of OAC were made according to guidelines or consensus or their own experience and even the fear of bleeding.

Guidelines or expert's consensus of nowadays is based on evidence derived from researches conducted out of China, especially mainland $(14,15)$. Therefore, there possibly exists a racial difference between patients from China and patients from other countries especially from Europe and
America. With this consideration, Chinese doctors are often reluctant to prescribe OAC to patients after a successful RFCA procedure. In fact, in our study, the success rate of paroxysmal AF catheter ablation was $89 \%$, which should cause the doctor's decision on discontinuation of oral anticoagulation. In specific, the main OAC used in China is warfarin which needs intensive monitoring international normalized ratio (INR) which is not convenient for most patients and patients' compliance is poor $(16,17)$.

Moreover, the reaction to warfarin differs significantly among Chinese patients (18). The same dose may not enough for one patient, but too much for another patient. As a result of all these reasons, warfarin is under-used in Chinese patients $(19,20)$. On the other hand, some patients were administrated with warfarin at a lower dosage without reaching the target INR, as described before (21).

Unfortunately, among 989 patients who stopped OAC during follow-up, 411 patients' $\mathrm{CHA}_{2} \mathrm{DS}_{2}$-VASc score was over 2. These patients were at higher risk of ischemic stroke/TIA before and after conversion to sinus rhythm. However, the actual risk of these patients after RFCA is still unclear. So, some doctors may not continue to prescribe OAC to patients after 3 months of the blank period after successful RFCA procedure $(22,23)$. In this study, we found that $2.6 \%$ of patients suffered stroke/TIA in those patients. This incidence was a little higher than that in patients who continued OAC therapy (26/989 vs. $11 / 502, \mathrm{P}=0.608)$ but not statistically. This result suggested that it is reasonable to continue to administrate $\mathrm{OAC}$ to patients with $\mathrm{CHA}_{2} \mathrm{DS}_{2}-$ VASc score over 2. However, this also raises a question of how to select patients really at high risk of ischemic stroke/ TIA because only 18 of 411 patients developed ischemic 
stroke/TIA. The incidence of SCI detected by MRI in patients who stopped OAC was lower than that in patients who did not. The main reason may be the lower $\mathrm{CHA}_{2} \mathrm{DS}_{2}-$ VASc score in patients who stopped OAC.

Another reason may be the under-use of OAC, especially warfarin. In this study, we found 3 -fold of patients who were administrated with warfarin had not reached the target INR during follow-up, and the reason for this phenomenon was described before. Some research suggested that RFCA could reduce the risk of cognition impairment or dementia $(24,25)$. RFCA procedure was related to SCI, and SCI was believed to be a risk factor of cognition impairment or dementia $(9,26,27)$. Theoretically, OAC can reduce the incidence of SCI, especially in patients with high risk (28). However, in those patients with continued OAC therapy, the incidence of SCI was a little high. The underlying reason for this is similar to the question mentioned above.

The incidence of major bleeding in patients who stopped OAC was significantly lower than that in patients who did not have bleeding with some patients who had not reached the INR target. Based on these conflicting facts, we suggest that further research should focus on how to precisely recognize the real patients at elevated risk of stroke, whether ischemic or hemorrhagic. For example, further study could investigate OAC therapy in patients with specific $\mathrm{CHA}_{2} \mathrm{DS}_{2}$-VASc scores, and especially the average score of 2-3. Alternatively, new techniques should be applied in precision medicine for this kind of patients.

In our study, we did not find the difference of death between two subgroups. The possible reason includes: the duration of follow-up was not long enough; the sample size was not big enough, and there was indeed no difference in mortality exist between two subgroups. Further study should also enroll more patients from more centers and follow up longer to figure out the real status of postablation anticoagulation and its influence on the prognosis of patients with paroxysmal AF.

\section{Acknowledgments}

Funing: None.

\section{Footnote}

Conflicts of Interest: All authors have completed the ICMJE uniform disclosure form (available at http://dx.doi. org/10.21037/apm-20-565). The authors have no conflicts of interest to declare.
Ethical Statement: The authors are accountable for all aspects of the work in ensuring that questions related to the accuracy or integrity of any part of the work are appropriately investigated and resolved.

Open Access Statement: This is an Open Access article distributed in accordance with the Creative Commons Attribution-NonCommercial-NoDerivs 4.0 International License (CC BY-NC-ND 4.0), which permits the noncommercial replication and distribution of the article with the strict proviso that no changes or edits are made and the original work is properly cited (including links to both the formal publication through the relevant DOI and the license). See: https://creativecommons.org/licenses/by-nc-nd/4.0/.

\section{References}

1. Guo Y, Tian Y, Wang H, et al. Prevalence, incidence, and lifetime risk of atrial fibrillation in China: new insights into the global burden of atrial fibrillation. Chest 2015;147:109-19.

2. Wang X, Fu Q, Song F, et al. Prevalence of atrial fibrillation in different socioeconomic regions of China and its association with stroke: Results from a national stroke screening survey. Int J Cardiol 2018;271:92-7.

3. Jagadish PS, Kirolos I, Khare S, et al. Post-operative atrial fibrillation: should we anticoagulate? Ann Transl Med 2019;7:407.

4. Calkins H, Hindricks G, Cappato R, et al. 2017 HRS/ EHRA/ECAS/APHRS/SOLAECE expert consensus statement on catheter and surgical ablation of atrial fibrillation. Europace 2018;20:e1-e160.

5. Calkins H, Kuck KH, Cappato R, et al. 2012 HRS/EHRA/ ECAS expert consensus statement on catheter and surgical ablation of atrial fibrillation: recommendations for patient selection, procedural techniques, patient management and follow-up, definitions, endpoints, and research trial design. J Interv Card Electrophysiol 2012;33:171-257.

6. Kirchhof P, Benussi S, Kotecha D, et al. 2016 ESC Guidelines for the management of atrial fibrillation developed in collaboration with EACTS. Eur Heart J 2016;37:2893-962.

7. Lévy S, Breithardt G, Campbell RW, et al. Atrial fibrillation: current knowledge and recommendations for management. Working Group on Arrhythmias of the European Society of Cardiology. Eur Heart J 1998;19:1294-320.

8. Ming J, Wei Y, Sun H, et al. Cost-Effectiveness of 
Cryoballoon Ablation Versus Radiofrequency Ablation for Paroxysmal Atrial Fibrillation in China: Results Based on Real-World Data.Value Health 2019;22:863-70.

9. Dong JZ, Sang CH, Yu RH, et al. Prospective randomized comparison between a fixed '2C3L' approach vs. stepwise approach for catheter ablation of persistent atrial fibrillation. Europace 2015;17:1798-806.

10. Rottner L, Bellmann B, Lin T, et al. Catheter Ablation of Atrial Fibrillation: State of the Art and Future Perspectives. Cardiol Ther 2020. doi: 10.1007/s40119-019-00158-2.

11. Yang WY, Du X, Jiang C, et al. The safety of discontinuation of oral anticoagulation therapy after apparently successful atrial fibrillation ablation: a report from the Chinese Atrial Fibrillation Registry study. Europace 2020;22:90-9.

12. Okumura Y, Nagashima K, Arai M, et al. Current Status and Clinical Outcomes of Oral Anticoagulant Discontinuation After Ablation for Atrial Fibrillation in Japan- Findings From the AF Frontier Ablation Registry. Circ J 2019;83:2418-27.

13. Själander S, Holmqvist F, Smith JG, et al. Assessment of Use vs Discontinuation of Oral Anticoagulation After Pulmonary Vein Isolation in Patients With Atrial Fibrillation. JAMA Cardiol 2017;2:146-52.

14. Atti V, Turagam MK, Viles-Gonzalez JF, et al. Anticoagulation After Catheter Ablation of Atrial Fibrillation: Is it time to Discontinue in Select Patient Population? J Atr Fibrillation 2018;11:2092.

15. Liang JJ, Elafros MA, Mullen MT, et al. Anticoagulation use and clinical outcomes after catheter ablation in patients with persistent and longstanding persistent atrial fibrillation. J Cardiovasc Electrophysiol 2018;29:823-32.

16. Sun $\mathrm{Y}, \mathrm{Hu} \mathrm{D}$, Stevens S, et al. Efficacy and safety of rivaroxaban versus warfarin in patients from mainland China with nonvalvular atrial fibrillation: A subgroup analysis from the ROCKET AF trial. Thromb Res 2017;156:184-90.

17. Wang C, Yang Z, Wang C, et al. Significant underuse of warfarin in patients with nonvalvular atrial fibrillation: results from the China national stroke registry. J Stroke Cerebrovasc Dis 2014;23:1157-63.

18. Wang ZZ, Du X, Wang W, et al. Long-Term Persistence of Newly Initiated Warfarin Therapy in Chinese Patients With Nonvalvular Atrial Fibrillation. Circ Cardiovasc Qual Outcomes 2016;9:380-7.

19. Wu J, Wang J, Jiang S, et al. The efficacy and safety of low intensity warfarin therapy in Chinese elderly atrial fibrillation patients with high CHADS2 risk score. Int J Cardiol 2013;167:3067-8.

20. Liang HF, Du X, Zhou YC, et al. Control of Anticoagulation Therapy in Patients with Atrial Fibrillation Treated with Warfarin: A Study from the Chinese Atrial Fibrillation Registry. Med Sci Monit 2019;25:4691-8.

21. Liu T, Hui J, Hou YY, et al. Meta-Analysis of Efficacy and Safety of Low-Intensity Warfarin Therapy for East Asian Patients With Nonvalvular Atrial Fibrillation. Am J Cardiol 2017;120:1562-7.

22. Freeman JV, Shrader P, Pieper KS, et al. Outcomes and Anticoagulation Use After Catheter Ablation for Atrial Fibrillation. Circ Arrhythm Electrophysiol 2019;12:e007612.

23. Gumbinger C, Holstein T, Stock C, et al. Reasons underlying non-adherence to and discontinuation of anticoagulation in secondary stroke prevention among patients with atrial fibrillation. Eur Neurol 2015;73:184-91.

24. Hsieh YC, Chen YY, Chien KL, et al. Catheter ablation of atrial fibrillation reduces the risk of dementia and hospitalization during a very long-term follow-up. Int J Cardiol 2020;304:75-81.

25. Jin MN, Kim TH, Kang KW, et al. Atrial Fibrillation Catheter Ablation Improves 1-Year Follow-Up Cognitive Function, Especially in Patients With Impaired Cognitive Function. Circ Arrhythm Electrophysiol 2019;12:e007197.

26. Gaita F, Anselmino M. Silent Cerebral Events During Catheter Ablation for Atrial Fibrillation: Not Yet to Be Forgotten. JACC Clin Electrophysiol 2018;4:1610-2.

27. Nakamura T, Okishige K, Kanazawa T, et al. Incidence of silent cerebral infarctions after catheter ablation of atrial fibrillation utilizing the second-generation cryoballoon. Europace 2017;19:1681-8.

28. Deneke T, Jais P, Scaglione M, et al. Silent cerebral events/ lesions related to atrial fibrillation ablation: a clinical review. J Cardiovasc Electrophysiol 2015;26:455-63.

Cite this article as: $\mathrm{Yu} \mathrm{R}, \mathrm{Xi} \mathrm{H}, \mathrm{Lu} J, \mathrm{Xu} F$, Shi L, Kong Q, Hu Y, Zhao X, Liu N. Real-world investigation on discontinuation of oral anticoagulation after paroxysmal atrial fibrillation catheter ablation in China. Ann Palliat Med 2020;9(3):940-946. doi: 10.21037/apm-20-565 